\title{
Harmonic oscillators in the Nosé-Hoover environment
}

\author{
V. L. Golo, ${ }^{1, *}$ Vl. N. Salnikov, ${ }^{1, \dagger}$ and K. V. Shaitan ${ }^{2, *}$ \\ ${ }^{1}$ Department of Mechanics and Mathematics, Moscow University, Moscow 119 899, Russia \\ ${ }^{2}$ Department of Biology, Moscow University, Moscow 119 899, Russia
}

(Received 16 February 2004; revised manuscript received 7 May 2004; published 29 October 2004)

\begin{abstract}
We study the dynamics of an ensemble of noninteracting harmonic oscillators in a nonlinear dissipative environment described by the Nosé-Hoover model, and find the histogram for energy regions of phase space against visiting time by employing numerical simulation. The results agree with the analysis of the NoséHoover equations effected with the method of averaging for small values of the dissipative parameter $\alpha$ of the thermostat. We find oscillations at frequencies proportional to $\sqrt{\alpha / m}, m$ being the characteristic mass of the particle, about the stationary state corresponding to equilibrium, for sufficiently small $\alpha$. In this region of $\alpha$ the histogram does not correspond to Gibbs' canonical distribution. For larger values of $\alpha$ the motion becomes irregular. The phenomena could have an important bearing upon simulating molecular dynamics in the NoséHoover thermostat.
\end{abstract}

DOI: 10.1103/PhysRevE.70.046130

PACS number(s): 02.70.Ns

\section{INTRODUCTION}

The Nosé-Hoover model [1-3] is widely used in molecular dynamics for simulating a system's behavior at constant temperature. The central idea of the model, which is the introduction of auxiliary dynamic variables to control kinetic energy, admits of various implementations. In the most simple and exploited form it amounts to considering a minimal nonlinear extension of the original equations for the system. It should be noted that Hamiltonian versions of the model drew considerable attention [4].

In the present paper we study the Nosé-Hoover model in the form generally employed in molecular dynamics, that is, a system constructed from initial Hamiltonian equations, i.e., using Newton's second law, by employing nonlinear dissipative terms on their right-hand sides, and an additional equation for the dissipation parameter, or "demon," $\gamma$, which is allowed to vary in time, so that the equations of evolution read

$$
\begin{gathered}
m_{i} \ddot{\vec{r}}_{i}=-\frac{\partial}{\partial \vec{r}_{i}} U\left(\vec{r}_{1}, \vec{r}_{2}, \ldots, \vec{r}_{N}\right)-\gamma \dot{\vec{r}}_{i}, \\
\dot{\gamma}=\alpha\left(\frac{2}{3 k_{b} T N} \sum_{i=1}^{N} \frac{m_{i} \vec{r}_{i}}{2}-1\right) .
\end{gathered}
$$

In this setting the Nosé-Hoover model is a Hamiltonian system with dissipation. For sufficiently small values of $\alpha$ the dissipative effects can be treated within the framework of perturbation theory.

Considerable criticism has been leveled at the NoséHoover approach (see [5] and references therein), because it runs across difficulties in providing the correct thermody-

\footnotetext{
*Electronic address: golo@mech.math.msu.su

†Electronic address: vladsal@moldyn.ru

"Electronic address: shaitan@moldyn.ru
}

namic behavior for simple harmonic systems; it is still generally accepted that its efficiency improves with an increase of complexity and dimension of the simulated system [5]. It should be noted that substantial improvement aimed at compatibility with Gibbs' ensembles has been effected on the original version of the Nosé-Hoover model (see the review article [6]). Indeed, one can reproduce Brownian motion by employing several thermostat variables, or demons, instead of the singular auxiliary $\gamma$ (see the comprehensive treatment of the subject in [6,7]). In this paper we study the initial version of the Nosé-Hoover model, which, from the point of view of dynamical theory, corresponds to a nonlinear timedependent dissipative environment and, therefore, has a physical interest of its own.

We focus our attention on ensembles of harmonic oscillators; the importance of such systems follows from the fact that among these is the harmonic lattice, familiar in the theories of solids and molecules. The potential energy of a harmonic lattice is given by the quadratic form

$$
U\left(\vec{r}_{1}, \vec{r}_{2}, \ldots, \vec{r}_{N}\right)=\sum_{i, j=1}^{N} \sum_{l, k=1}^{3} \lambda_{i j}^{l k} r_{i} r_{j}^{k}
$$

in which $r_{i}^{l}$ is the $l$ th coordinate of the $i$ th particle. To see the symmetry properties of the model we may cast Eq. (1) in the matrix form

$$
m_{i} \ddot{r}_{i}+(\Lambda r)_{i}+\gamma \dot{r}_{i}=0
$$

in which $\Lambda$ is the matrix of force constants $\lambda_{i j}^{l k}$. It can be transformed by an appropriate orthogonal transformation $R$ to the diagonal form

$$
U\left(w_{1}, w_{2}, \ldots, w_{3 N}\right)=\sum_{i=1}^{3 N} \lambda_{i} w_{i}^{2} .
$$

Assume that all $m_{i}$ are equal, $m_{i}=m$, and let $R$ be the matrix of the orthogonal transformation mentioned above, and $w$ a $3 \mathrm{~N}$-dimensional vector of coordinates with respect to the new coordinate system determined by $R$. Since $R(t)=R$ is 
constant, and $\gamma$ is an invariant of the orthogonal transformation, we may cast Eq. (1) in the form

$$
R \ddot{w}+\Lambda R w+\gamma R \dot{w}=0,
$$

so that the equation acquires the form

$$
\ddot{w}+\left(R^{T} \Lambda R\right) w+\gamma \dot{w}=0
$$

in which the matrix $R^{T} \Lambda R$ is the diagonal one.

It should be noted that three of the eigenvalues $\lambda_{i}$ are equal to zero, which corresponds to the conservation of momentum of the lattice. In what follows, we shall neglect the modes of zero frequencies, considering only internal degrees of freedom corresponding to thermodynamical properties of the system.

Thus, we have transformed the original problem of harmonic lattice to that for a set of harmonic oscillators, which do not interact with each other. It is more tractable from an analytical point of view. At this point it should be noted that molecular dynamics usually involves nonlinear systems, and therefore it is of interest to investigate possible nonlinear extensions of our results. For the moment we may only claim that, according to the numerical simulation, very small nonlinear terms in Eq. (1) do not lead to any drastic changes, but the problem needs thorough study, which is to be found elsewhere.

At this point it is worthwhile to notice that problems of molecular dynamics involve dynamical systems of extremely high dimension, and this circumstance brings about specific difficulties for numerical simulations. The best approach to the problem is to use analytical methods in conjunction with the numerical ones, e.g., for the Nosé-Hoover model, where systematic investigation of high-dimensional cases is particularly interesting (see [5]). To this end we use the method of "windows" worked out for the needs of relaxation dynamics of spin in superfluid ${ }^{3} \mathrm{He}$ (see the review article [8]) to obtain a general picture of the Nosé-Hoover dynamics for an ensemble of harmonic oscillators. We show that, if the dissipative constant $\alpha$ is small enough, the dynamics is characterized by the presence of oscillations around the stationary solution corresponding to an equilibrium for which the phase-space sampling is different from the normal law. In fact, we find that for sufficiently large $\alpha$ the oscillations disappear, and the motion of the system becomes irregular.

\section{STOCHASTIC PROPERTIES OF THE SYSTEM}

The numerical analysis of the Nosé-Hoover model given by Eq. (1), which is a high-dimensional nonlinear dissipative system, is a serious challenge, and, in fact, it is generally confined to low-dimensional situations (see [9], in which the case of two oscillators is considered). In treating highdimensional problems the key point is the wise choice of output variables. In the present case it is dictated by the physics of the problem, and taking into account the structure of the Nosé-Hoover model, that is, its being a Hamiltonian system with dissipation, we employ to that effect the total energy of the system $E$,

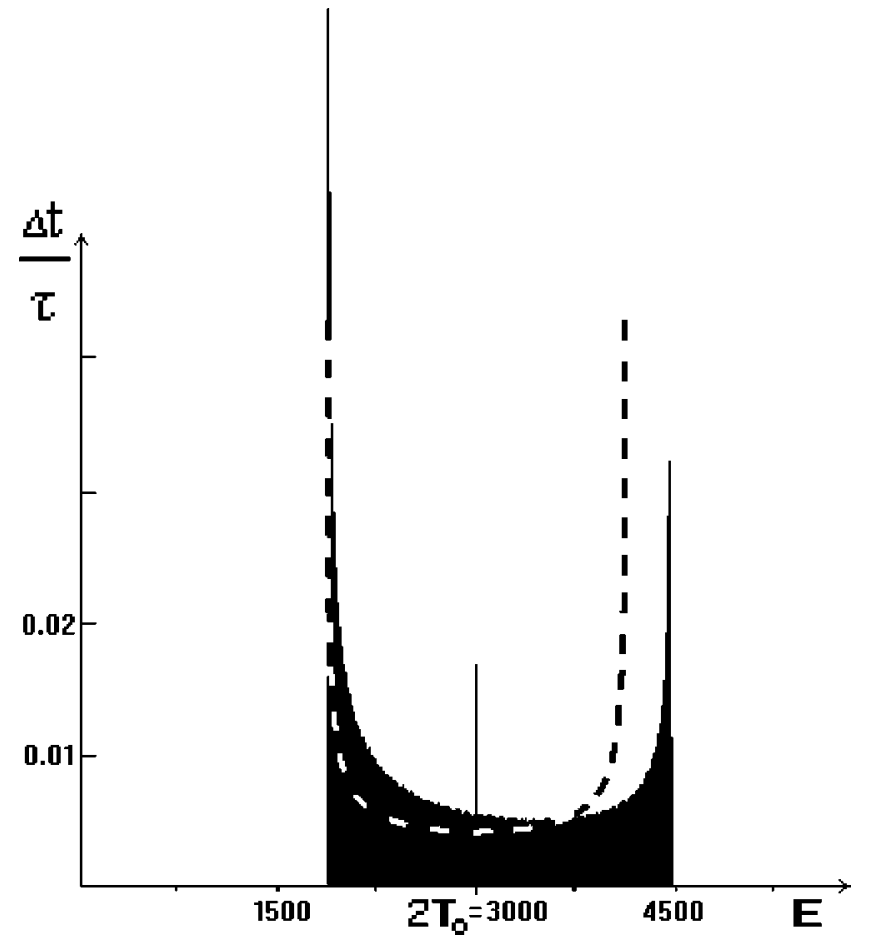

FIG. 1. Energy regions of phase space against visiting time. Number of oscillators $N=1000$, dissipative parameter $\alpha=0.01$, temperature parameter $T_{R}=3, T_{0}=0.5 N T_{R}$; all frequencies $\omega_{i}=1$; initial coordinates $x_{i}$ and momenta $p_{i}$ distributed randomly from -2 to +2 ; initial $\gamma=0$. Averaged distribution, Eq. (15), given by dashed line.

$$
E=E_{\mathrm{kin}}+U,
$$

and the dissipative variable $\gamma$. Directing the output in the $(E-\gamma)$ plane, we obtain a kind of two-dimensional window on the phase space of the model, which has dimension $2 N+1$, where $N$ is the number of oscillators. Since we aim at studying situations in which $N$ is large, the two-dimensional reduction is of primary importance. Next, one should look for the distribution law of the the system in phase space, and one could expect that it should be either the microcanonical or the Gibbs one; the first is characterized by its being centered on a particular value of energy $E_{0}$, whereas the latter has a characteristic bell shape. We shall see that the actual situation is richer (Fig. 1).

It is worthwhile to employ units of physical quantities corresponding to the scales of molecular dynamics. In our computer calculations we shall use the unit of mass $\approx 10^{-23} \mathrm{~g}$, the unit of length $10^{-8} \mathrm{~cm}$, and the unit of time $2 \times 10^{-13} \mathrm{~s}$, so that the unit of energy $\mathcal{E}$ reads

$$
\mathcal{E}=1.25 \times 10^{-14} \mathrm{erg},
$$

and we may introduce the reduced temperature

$$
T_{R}=\frac{k_{b} T}{\mathcal{E}},
$$

which, at room temperatures, turns out to be of the order 1.

To find the distribution law we consider the partition of the phase space into regions 


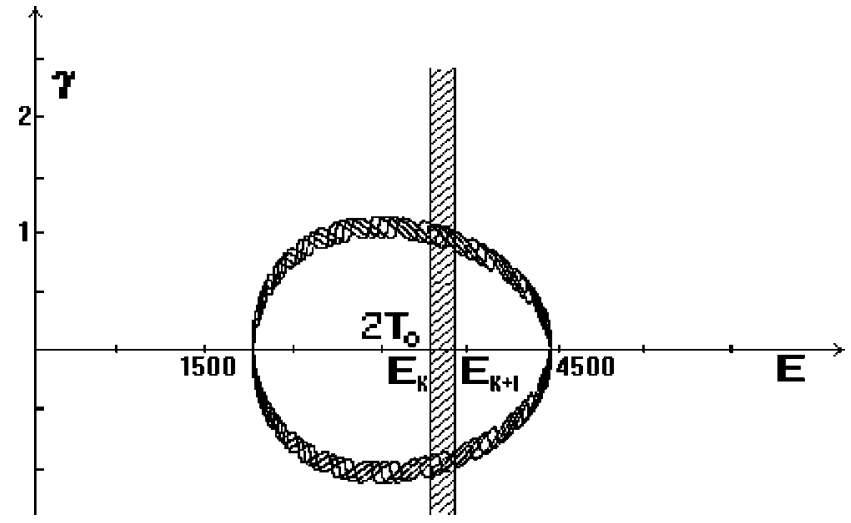

FIG. 2. Trajectory in $(E-\gamma)$ window. Regions $\mathcal{R}_{k}$ indicated by shaded vertical band. Values of parameters and initial data are the same as in Fig. 1.

$$
\mathcal{R}_{1}, \mathcal{R}_{2}, \ldots, \mathcal{R}_{k}, \ldots, \mathcal{R}_{L}
$$

corresponding to the energy intervals $E_{k} \leqslant E \leqslant E_{k+1}$, assumed to be of equal size, and compile a record of periods of time $t_{1}, t_{2}, \ldots, t_{k}, \ldots, t_{L}$ which the system spends in regions (4); the total time of simulation reads

$$
\tau=\sum_{i=1}^{L} t_{i}
$$

The frequencies for the system's visiting the regions are given by the equation

$$
p_{k}=\frac{t_{k}}{\tau}, \quad k=1,2, \ldots, L
$$

It is convenient to use a representation for the set of frequencies by a histogram, that is, rectangles whose widths represent the energy intervals (4) and whose heights represent the corresponding frequencies. It is worth noting that the partition of the phase space into the energy regions (4) can be effected in a more graphic form with the help of the $(E-\gamma)$ window on the phase space. In fact, the numerical simulation gives a picture of the system's motion, the times $t_{k}$ being those spent in the bands determined by the constraints $E_{k}$ $\leqslant E \leqslant E_{k+1}$, as is illustrated in Fig. 2.

To be specific, we consider a set of one-dimensional harmonic oscillators given by the Hamiltonians

$$
\mathcal{H}_{i}=\frac{p_{i}^{2}+m^{2} \omega_{i}^{2} x_{i}^{2}}{2 m}, \quad i=1,2, \ldots, N .
$$

The oscillators do not interact with each other, so that the total Hamiltonian reads

$$
\mathcal{H}=\mathcal{H}_{1}+\mathcal{H}_{2}+\cdots+\mathcal{H}_{N}
$$

The choice of the dissipative parameter $\alpha$ is important. In accord with the general prescription of molecular dynamics [10], one usually takes it small. It is a matter of a certain interest to see what happens if $\alpha$ is of intermediate size. We come to the conclusion that the actual small size of $\alpha$ is to be gauged in accord with the smallest of frequencies $\omega_{1}, \omega_{2}, \ldots, \omega_{N}$,

$$
\omega_{L}=\min \left\{\omega_{1}, \omega_{2}, \ldots, \omega_{N}\right\} .
$$

In fact, our numerical calculations, as well as the averaging method (see Sec. III) indicate that the region of really small $\alpha$ should comply with the requirement

$$
\Omega_{\alpha}<\omega_{L}
$$

in which

$$
\Omega_{\alpha}=\sqrt{\frac{\alpha}{m}} .
$$

One may state that the characteristic frequency $\Omega_{\alpha}$ should be below the lowest edge of the oscillator band.

Therefore, our calculations can be partitioned into two groups: F1, long time scales for which condition (7) is satisfied; F2, intermediate time scales for which (7) is broken and the characteristic frequencies lie inside or above the oscillator band $\omega_{1}, \omega_{2}, \ldots, \omega_{N}$.

Let us consider first case F1, which strictly follows the molecular dynamics prescription [10]. In accord with Eq. (4) we obtain the histogram given in Fig. 1, describing the probability distribution $\rho$ for the system in phase space. It is quite different from the microcanonical or Gibbs distribution. It is important that the window $(E-\gamma)$ provides a means for elucidating the form of the fluctuations around the stationary state given by the equations

$$
\gamma=0, \quad E=2 T_{0}, \quad E_{\text {kin }}=T_{0}
$$

for some value $T_{0}$ of the temperature parameter [cf. Eq. (13)]. The circular motion seen in the $(E-\gamma)$ window is characterized by a mean angular velocity given by the law $\sqrt{\alpha / m}$ to within one-thousandth, that is, we obtain $\Omega_{\alpha}$ indicated above. The fact agrees with the histogram given in Fig. 1 (see Sec. III for the details).

The width of the ring swept over by a trajectory as seen in the $(E-\gamma)$ window depends on the structure of the oscillator band. If the scattering of the oscillator frequency is small, for example, they are all equal, the width is sufficiently pronounced (see Fig. 2). For the case of a harmonic lattice when the frequencies read

$$
\omega_{k}^{2}=\frac{4 C}{m} \sin ^{2} \frac{2 \pi k a}{N}
$$

the width is very small (see Fig. 3). The same phenomenon also takes place for a sufficiently dense random distribution of the oscillators. For the case of an ideal gas the oscillation law $\sqrt{\alpha / m}$ was found in [9] [see Eqs. (5) and (8) of [9]].

The results of the calculation can be cast in the form of a histogram, and it should be noted that the shape of the histogram depends on the amplitude of the oscillations, that is, the size of deviations in the energy $E$ from the stationary value $2 T_{0}$ determined by the temperature parameter of the model. In the next section we shall find the distribution using an averaging method for $\alpha$ which satisfies the requirement $\Omega_{\alpha}<\omega_{L}$, and it is worth noting that the numerical results are in good agreement with those given by the averaging, as is seen from Fig. 4. The energy distribution that should correspond to Gibbs' canonical ensemble for the set of harmonic 


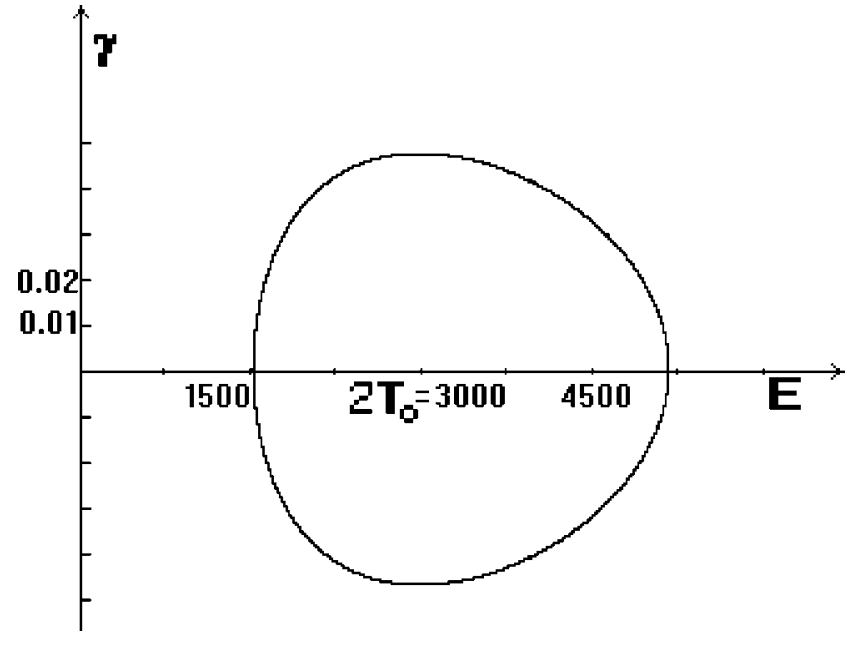

FIG. 3. Very narrow band swept by a trajectory in $(E-\gamma)$ window. Number of oscillators $N=1000$; dissipative parameter $\alpha$ $=10^{-5}$; temperature parameter $T_{R}=3, T_{0}=0.5 N T_{R}$; frequencies $\omega_{i}$ given by Eq. (8) with $4 C / m=2.25, a=1$; initial coordinates $x_{i}$ and momenta $p_{i}$ distributed randomly from -2 to +2 ; initial $\gamma=0$.

oscillators at temperature $T$ is given by the equation

$$
d \rho=c_{N} \frac{E^{N-1}}{\left(2 \pi k_{b} T\right)^{N}} e^{-E / k_{b} T} d E
$$

and is totally different from Fig. 1. The discrepancy between the obtained and expected distributions indicates that the Nosé-Hoover model describes a kind of nonlinear dissipative system having special properties.

Turn now to case F2. A typical situation is illustrated in Fig. 5. We see that a trajectory seen in the $(E-\gamma)$ window

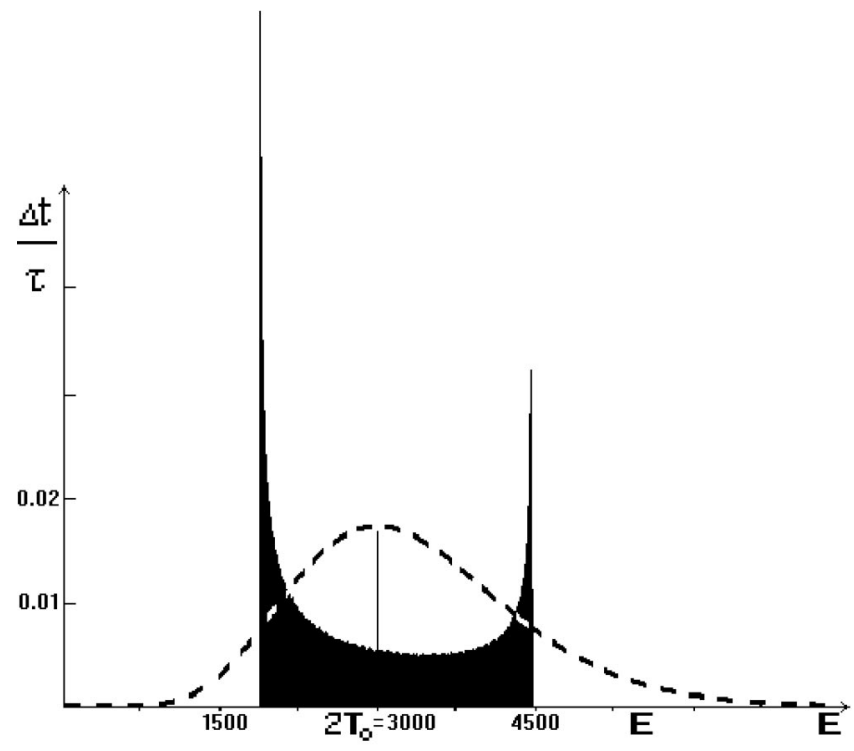

FIG. 4. Characteristic frequency $\Omega_{\alpha}$ below oscillator frequency band. Values of parameters and initial data are the same as in Fig. 1. Filled area gives energy distribution against visiting time according to numerical simulation; dashed line corresponds to Gibbs distribution.

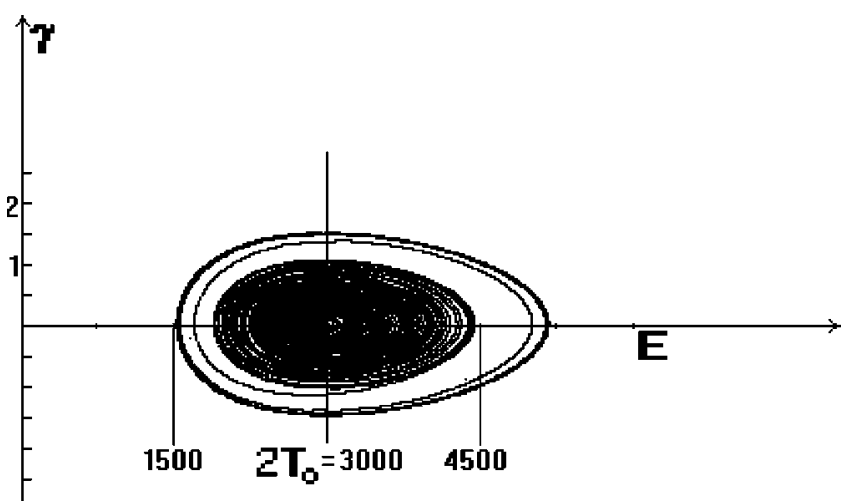

FIG. 5. Trajectory in window $(E-\gamma)$. Characteristic frequency $\Omega_{\alpha}$ inside oscillator frequency band. Number of oscillators $N$ $=1000$; dissipative parameter $\alpha=0.01$; temperature parameter $T_{R}$ $=3, T_{0}=0.5 N T_{R}$; frequencies $\omega_{i}$ given by Eq. (8) with $4 \mathrm{C} / \mathrm{m}$ $=2.25, a=1$; initial coordinates $x_{i}$ and momenta $p_{i}$ distributed randomly from -2 to +2 .

sweeps over a region in the $E-\gamma$ plane. The motion is quite irregular and needs specific study, which is to be done elsewhere. The energy distribution illustrated in Fig. 6 strongly suggests that it is chaotic. It should be noted that in case F2 the averaging method of Sec. III breaks down, for values of $\alpha$ not small enough. But we cannot claim that the histogram corresponds to the Gibbs distribution. In fact, its shape strongly depends on the data, i.e., oscillator frequencies, initial data, and values of $\alpha$. This circumstance could be instrumental in the use of the Nosé-Hoover model for molecular dynamics, for one might obtain helpful distributions by adjusting the model's data.

\section{AVERAGED SYSTEM}

The simulation of the last section, which was thoroughly checked by calculating with different algorithms and comparing their results, might nonetheless be subject to artifacts and errors. In this respect, it is important that analytical means capable of verifying Sec. II have been used and resulted in agreement with the numerical work.

Let us notice that according to the prescription described in the Introduction [see Eq. (1)] the ensemble of $N$ harmonic

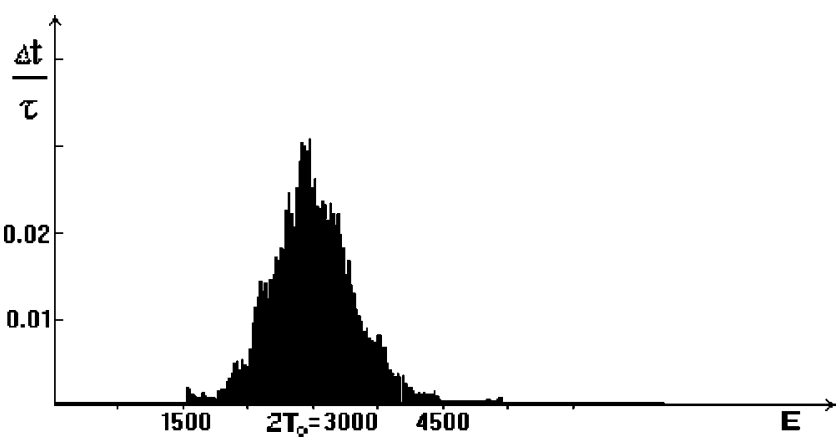

FIG. 6. Energy regions of phase space against visiting time; characteristic frequency $\Omega_{\alpha}$ inside the oscillator band. The data are the same as in Fig. 5. 
oscillators confined to the Nosé-Hoover thermostat is described by the system of equations

$$
\begin{gathered}
\ddot{x}_{i}+\omega_{i}^{2} x_{i}=-\frac{\gamma}{m_{i}} \dot{x}_{i} \quad(i=1,2, \ldots, N), \\
\dot{\gamma}=\alpha\left(\frac{2}{k_{b} T N} \sum_{i=1}^{N} \frac{m_{i} \dot{x}_{i}^{2}}{2}-1\right)
\end{gathered}
$$

in which all dimensional quantities are in the CGS system of units. It is important that the dissipative constant $\alpha$ is suggested to be small, $\alpha \ll 1$, or, to be more precise, $\Omega_{\alpha}$ $\ll \omega_{L}, \omega_{L}$ being the lowest edge of the oscillator band. In fact, we shall require that the characteristic frequencies satisfy condition (7). The structure of the oscillator frequency set deserves special attention. If the number of oscillators is large, the frequencies may be very dense, so that one may regard them as a band, as may happen, for example, in the case of numerical modeling of large molecules. At the same time, even for large systems there may exist situations in which the number of different values of $\omega_{i}$ is small, even all of them being equal. Our analysis, based on the averaging method, depends on condition (7) being satisfied, that is, small values of $\alpha$, usually employed in molecular dynamics (see [10]). If (7) is broken, the nature of the system's dynamics changes drastically, and one may expect its becoming chaotic, as could be expected from Figs. 5 and 6. This phenomenon may be useful for the practical needs of molecular dynamics; its detailed treatment is to be found elsewhere.

Thus, we may say that $\Omega_{\alpha}$ should lie below the edge of the band determined by the oscillators' frequencies and correspond to a time scale larger than the time scales of the oscillators. This assumption enables us to choose, as a first approximation, the nondissipative regime for which $\alpha$ $\equiv 0, \gamma=0$, and there is an exact solution given by the equation

$$
x_{i}=A_{i} \cos \left(\omega_{i} t+\phi_{i}\right), \quad \dot{x}_{i}=-A_{i} \omega_{i} \sin \left(\omega_{i} t+\phi_{i}\right) .
$$

The energy of the $i$ th oscillator divided by mass $m$ reads

$$
e_{i}=\frac{\dot{x}_{i}^{2}}{2}+\frac{\omega_{i}^{2} x_{i}^{2}}{2}=\frac{A_{i}^{2} \omega_{i}^{2}}{2} .
$$

The masses of the particles are assumed to be equal, $m_{i}=m$, the oscillators being allowed to differ only in their frequencies $\omega_{i}$. Equations (11), from the topological point of view, mean that the ensemble's motion belongs to an $N$-dimensional torus, the whole phase space being foliated by the tori. We shall consider the system at temperature $T$. Since the parameter $\alpha$ is small, we may take into account the nonlinear dissipative terms on the right-hand sides of Eqs. (10), within the framework of the averaging approach, that is, by substituting the basic equations (11) into the right-hand sides of the exact equations,

$$
\dot{e}_{i}=-\frac{\gamma}{m}\left(\frac{A_{i}^{2} \omega_{i}^{2}}{2}-\frac{A_{i}^{2} \omega_{i}^{2}}{2} \cos \left(2 \omega_{i} t+2 \phi_{i}\right)\right),
$$

$$
\dot{\gamma}=\alpha\left(\frac{m}{2 T_{*}} \sum_{i=1}^{N} \frac{A_{i}^{2} \omega_{i}^{2}}{2}-1\right),
$$

in which

$$
T_{*}=\frac{1}{2} N k_{b} T
$$

and we cancel out the oscillating terms. The procedure means, as was claimed above, that we consider a time scale larger than the largest period of our oscillators, or, to put it the other way round, the frequencies of the averaged system should be lower than the band determined by the oscillators' frequencies. Thus, we obtain the averaged equations

$$
\begin{gathered}
\dot{e}_{i}=-\frac{\gamma}{m} \frac{A_{i}^{2} \omega_{i}^{2}}{2}, \\
\dot{\gamma}=\alpha\left(\frac{m}{2 T_{*}} \sum_{i=1}^{N} \frac{A_{i}^{2} \omega_{i}^{2}}{2}-1\right),
\end{gathered}
$$

or

$$
\dot{e}_{i}=-\frac{\gamma}{m} e_{i}, \quad \dot{\gamma}=\alpha\left(\frac{m}{2 T_{*}} \sum_{i=1}^{N} e_{i}-1\right),
$$

as follows from Eq. (12).

Since the total energy of the system is given by the equation

$$
E=m \sum_{i=1}^{N} e_{i}
$$

we obtain the following two equations:

$$
\dot{E}=-\frac{\gamma}{m} E, \quad \dot{\gamma}=\frac{\alpha}{2 T_{*}}\left(E-2 T_{*}\right),
$$

which have a stationary solution describing the oscillators at the temperature parameter $T_{*}$, given by Eq. (13), or, using the units of Sec. II, $E=2 T_{0}$,

$$
E=2 T_{*}, \quad \gamma=0 .
$$

Close to the stationary solutions we have the equations for energy

$$
E=2 T_{*}+Z .
$$

Therefore the equations for $Z$ and $\gamma$ acquire the forms

$$
\dot{Z}=-\frac{\gamma}{m} Z-2 \frac{\gamma}{m} T_{*}, \quad \dot{\gamma}=\frac{\alpha}{2 T_{*}} Z .
$$

On linearizing the equations indicated above, we obtain

$$
\dot{Z}=-\frac{2 T_{*}}{m} \gamma, \quad \dot{\gamma}=\frac{\alpha}{2 T_{*}} Z,
$$

and hence the equation for $Z$ 


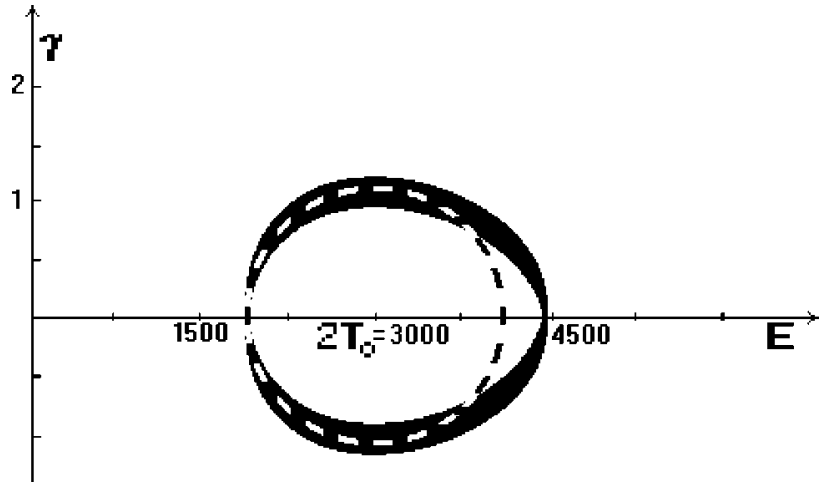

FIG. 7. Oscillations round stationary solution. Numerical simulation corresponds to filled area, averaged equations to dashed line. The data are the same as in Fig. 1.

$$
\ddot{Z}+\frac{\alpha}{m} Z=0
$$

which has the form of a harmonic oscillator with the frequency

$$
\sqrt{\frac{\alpha}{m}}
$$

Solutions to Eqs. (10) are illustrated in the $(E-\gamma)$ plane, in Fig. 7.

We may use Eq. (14) to find the time spent by the system in the region of the phase space corresponding to the energy interval $E_{1}<E<E_{2}$. In fact, from the periodic motion of the system, we infer that the visiting frequency $\Delta t / t$, in the averaged approximation, reads

$$
\frac{\Delta t}{t}=\left[\arcsin \frac{E_{2}-2 T_{*}}{E_{\max }-2 T_{*}}-\arcsin \frac{E_{1}-2 T_{*}}{E_{\max }-2 T_{*}}\right] .
$$

It is convenient to use the density of the visiting events per energy interval,

$$
\begin{aligned}
\lim _{E_{2}-E_{1} \rightarrow 0}\left(\frac{1}{E_{2}-E_{1}} \frac{\Delta t}{t}\right) & =\frac{1}{2 \pi} \frac{d}{d E} \arcsin \frac{E-2 T_{*}}{E_{\max }-2 T_{*}} \\
= & \frac{1}{2 \pi\left(E_{\max }-2 T_{*}\right)} \\
& \times\left[1-\left(\frac{E-2 T_{*}}{E_{\max }-2 T_{*}}\right)^{2}\right]^{-1 / 2} .
\end{aligned}
$$

The cusp form of the dashed line for the averaged distribution in Fig. 1 is in agreement with the singularity at $E$ $=E_{\max }$ in the above equation.

\section{CONCLUSION}

We have studied the dynamics of an ensemble of harmonic oscillators confined to the Nosé-Hoover thermostat, the number of oscillators $N$ being large, and the dissipative constant $\alpha$ small. Our analysis indicates that the nonlinear dynamics of the model has very interesting specific features even in the case of a simple harmonic system, and in this respect the characteristic frequency of collective oscillations $\Omega_{\alpha}=\sqrt{\alpha / m}$ deserves particular attention. Indeed, if the mass $m$ of a particle, corresponding to the oscillator, is $\approx 10^{-23} \mathrm{~g}$, and $\alpha \sim 0.1 \mathrm{~g} / \mathrm{s}^{2}$, the frequency of the oscillations generated by the thermostat dynamics should be in the region of 100 $\mathrm{GHz}$, that is, the low-frequency region of molecular vibrations. At the same time it is worth noting that the NoséHoover model corresponds to a Hamiltonian system confined to a dissipative environment, that is, it comprises a base Hamiltonian system, e.g., oscillators, and a dissipative extension formed by auxiliary variables; in the present case it is the variable $\gamma$. A similar system, even though more sophisticated, is the Leggett-Takagi theory of spin dynamics in superfluid phases of helium-3 [11,12], in which the equations describing the spin motion are augmented by an equation for the order parameter that contains a dissipative term. The situation is reminiscent of that taking place in the hydrodynamical treatment of viscous phenomena in the $\mathrm{GHz}$ region, where, according to the theory worked out by Mandelstam and Leontovic (see [13]), the effects of dissipation can be accommodated by employing an auxiliary dynamical variable $\xi$, which describes certain states of the system, e.g., the concentration of a chemical reagent. The evolution equations for $\xi$ have dissipative character, for they should describe the system's coming to equilibrium, although the initial equations for the system could be of Hamiltonian form. The Nosé-Hoover model may turn out to be of a similar kind and thus helpful in studying interesting physical problems.

It is also worth noting that for very large systems, for example, macromolecules, the lowest edge of the oscillator band may turn out to be very small, as is suggested by the dispersion law for a harmonic lattice, given by Eq. (8), which describes the dispersion of elastic waves within the Bornvon Kármán approximation, i.e., imposing periodic boundary conditions. The error with respect to the dispersion of the free lattice is proportional to the ratio of surface sites to the total number of lattice sites (see [14]). We see that the lowest edge of the frequency band for large $N$ is very small, and the application of the Nosé-Hoover thermostat should require intermediate values of the dissipative parameter $\alpha$, so that the characteristic frequency $\Omega_{\alpha}$ should lie inside the frequency band of the lattice, and the motion might become chaotic. These phenomena may turn out to be useful for the needs of molecular dynamics.

\section{ACKNOWLEDGMENTS}

We are thankful to W. G. Hoover and A. Tenenbaum for useful correspondence. This work was supported by the NS Grant no. 1988.2003.1, and RFFI Grants No. 01-01-00583, No. 03-02-16173, and No. 04-04-49645. 
[1] W. G. Hoover, Time Reversibility, Computer Simulations and Chaos (World Scientific, Singapore, 2001), Chap. 2.

[2] S. Nosé, Prog. Theor. Phys. Suppl. 103, 1 (1991).

[3] W. G. Hoover and B. L. Holian, Phys. Lett. A 211, 253 (1996)

[4] W. G. Hoover, Phys. Rev. A 31, 1695 (1985).

[5] M. D’Alessandro, A. Tenenbaum, and A. Amadei, J. Phys. Chem. B 106, 5050 (2002).

[6] W. G. Hoover, K. Aoki, C. G. Hoover, and S. V. DeGroot, Physica D 187, 253 (2004).

[7] D. Kuznecov, A. Bugac, and W. Bauer, Ann. Phys. (N.Y.) 204, 155 (1990).

[8] V. L. Golo, Int. J. Shape Model. 1, 109 (1994).
[9] H. A. Posch, W. G. Hoover, and F. J. Vesely, Phys. Rev. A 33, 4253 (1985).

[10] J. Elliot (unpublished).

[11] A. J. Leggett and S. Takagi, Ann. Phys. (N.Y.) 106, 79 (1977).

[12] V. L. Golo and A. A. Leman, in Helium Three, edited by W. P. Halperin and L. P. Pitaevskii (Elsevier, Amsterdam, 1990).

[13] L. D. Landau and E. M. Lifshitz, Hydrodynamics (Nauka, Moscow, 1988), Chap. 8.

[14] A. A. Maradudin, E. W. Montroll, and G. E. Weiss, Theory of Lattice Dynamics in the Harmonic Approximation (Academic, New York, 1963), Chap. 3. 